\title{
Estudo da qualidade das águas subterrâneas de abastecimento em bairros na cidade de parauapebas a partir de parâmetros físico- químicos
}

Amostras de águas subterrâneas foram coletadas em 20 locais de amostragem distribuídos em nove bairros da Cidade de Parauapebas (Estado do Pará, Brasil), em 2019, com o objetivo de avaliar a qualidade das águas subterrâneas captadas em poços tubulares potencialmente utilizados para consumo humano. Para todas as amostras de águas subterrâneas foram analisados nove parâmetros físico-químicos, tais como temperatura $(T)$, potencial hidrogeniônico $(\mathrm{pH})$, condutividade elétrica (CE), turbidez (TRB), cor , teor de cloreto (Cl-) e sólidos totais dissolvidos (STD), com a intenção de contribuir para o controle da qualidade de potabilidade das águas subterrâneas de acordo com os limites estabelecidos pelo Ministério da Saúde (Portaria № 2914/2011) e Conselho Nacional de Meio Ambiente (Resolução № 396/2008). Os parâmetros físico-químicos estudados mostraram que nas águas subterrâneas coletadas em poços tubulares no bairro Da Paz e foram encontrados os valores mais altos de sólidos totais dissolvidos $(175,0$ a $271,8 \mathrm{mg} \mathrm{L}-1)$, condutividade elétrica $(136,3$ a $216,7 \mu \mathrm{S} \mathrm{cm}-1)$ e cor $(5,0$ a 15,0 uH), servindo de indicativos para identificação de possíveis atividades antropogênicas de contaminação na região, principalmente em relação à descarga de efluentes domésticos. Os resultados obtidos dos parâmetros físico-químicos para todas as amostras se apresentaram abaixo dos limites estabelecidos de potabilidade para águas subterrâneas de acordo com a legislação Brasileira.

\section{Study of groundwater quality supply in neighborhoods from the parauapebas city based on physicochemical parameters}

\begin{abstract}
Groundwater were sampled at 20 sampling locations of nine nighborhoods of Parauapebas City (Pará State, Brazil) in 2019, with objective of evaluate the quality of groundwater sampled in tubular wells potentially used for human consumption. For all groundwater samples were analyzed nine physicochemical parameters, such as temperature $(T)$, hydrogen potential $(\mathrm{pH})$, electrical conductivity (EC), turbidity (TRB), color, chloride content (Cl-) and total dissolved solids (TDS), with the intention of contributing to the control of the quality of portability groundwater in accordance with the limits established by Ministério da Saúde (Portaria № 2914/2011) and the Conselho Nacional de Meio Ambiente (Resolução № 396/2008). The physicochemical parameters studied showed that in the groundwate collected in tubular wells in the neighborhood of Da Paz, the highest values of total dissolved solids (175.0 to 271.8 mg L-1), electrical conductivity (136.3 at 216.7 $\mu \mathrm{S} \mathrm{cm}-1$ ) and color (5.0 to $15.0 \mathrm{uH}$ ), serving as indicators for the identification of possible anthropogenic contamination activities in the region, mainly in relation to the discharge of domestic effluents. The results obtained from the physicochemical parameters for all samples were below the established limits of potability for groundwater in accordance with Brazilian legislation.
\end{abstract}

Keywords: Subterranean water; Physical-Chemical Parameters; Potability Quality.

Topic: Engenharia de Recursos Hídricos

Reviewed anonymously in the process of blind peer.
Received: 18/03/2020

Approved: 20/04/2020
Henrique Sousa Chaves (DD

Universidade Federal Rural da Amazônia, Brasil

http://lattes.cnpq.br/2142593099019750 http://orcid.org/0000-0002-6754-0576 hs.chaves123@gmail.com

Denilton Galvão de Morais Universidade Federal Rural da Amazônia, Brasil

http://lattes.cnpq.br/5648851496617628 denilton475@gmail.com

Karen Albuquerque Dias da Costa (iD Universidade Federal do Pará, Brasil http://lattes.cnpq.br/8493677235656139 http://lattes.cnpq.br/8493677235656139 karenquimica123@yahoo.com.br
Igor Vinícius de Oliveira (iD) Universidade Federal do Sul e Sudeste do Pará, Brasil

http://lattes.cnpq.br/1133025899150852 http://orcid.org/0000-0003-4218-5587 igor.oliveira@unifesspa.edu.br

Kelly das Graças Fernandes Dantas (id Universidade Federal do Pará, Brasil http://lattes.cnpq.br/72277777727553334 http://orcid.org/0000-0002-5424-2908 kdgfernandes@ufpa.br

\section{Claude Rosa da Silva}

Universidade Federal Rural da Amazônia, Brasil http://lattes.cnpq.br/5005233180543061 clararoze@yahoo.com.br

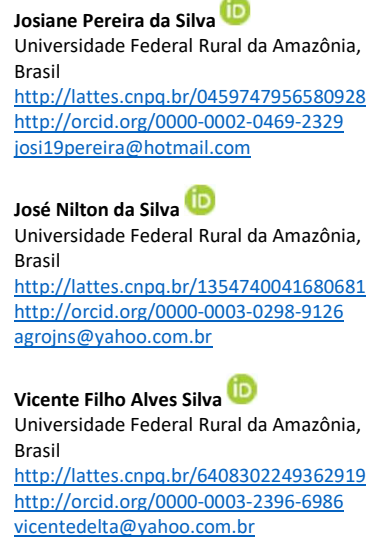
Brasil

http://lattes.cnpq.br/0459747956580928 http://orcid.org/0000-0002-0469-2329 josi19pereira@hotmail.com

osé Nilton da Silva

Universidade Federal Rural da Amazônia, Brasil

http://lattes.cnpq.br/1354740041680681 http://orcid.org/0000-0003-0298-9126 agroins@yahoo.com.br

Vicente Filho Alves Silva (ib Universidade Federal Rural da Amazônia, Brasil

http://lattes.cnpq.br/6408302249362919 http://orcid.org/0000-0003-2396-6986 vicentedelta@yahoo.com.br

Priscilla Andrade Silva (D)

Universidade Federal Rural da Amazônia, Brasil

http://lattes.cnpq.br/7666887041806711 http://orcid.org/0000-0002-2774-3192 prisciandra@yahoo.com.br

\section{Fábio Israel Carvalho (iD}

Universidade Federal Rural da Amazônia, Brasil

http://lattes.cnpq.br/8221002637257793 http://orcid.org/0000-0002-8995-2141 fabioimc@yahoo.com.br

Referencing this:

CHAVES, H. S.; MORAIS, D. G.; COSTA, K. A. D.; OLIVEIRA, I. V.; DANTAS, K. G. F.; SILVA, C. R.; SILVA, J. P.; SILVA, J. N.; SILVA, V. F. A.; SILVA, P. A.; CARVALHO, F. I.. Estudo da qualidade das águas subterrâneas de abastecimento em bairros na cidade de parauapebas a partir de parâmetros físico-químicos. Revista Ibero Americana de Ciências Ambientais, v.11, n.3, p.113-121, 2020. DOI: http://doi.org/10.6008/CBPC2179-6858.2020.003.0011 


\section{INTRODUÇÃO}

A água é essencial para vida e embora seja encontrada em maior abundância no planeta, a sua disponibilidade é restrita, pois apenas $0,8 \%$ da água do planeta está disponível para o consumo humano. A contaminação é um fator que contribui para diminuir sua disponibilidade, em função disto, as reservas disponíveis no subsolo vêm sendo cada vez mais exploradas (PANERO et al., 2008).

O comprometimento da qualidade das águas subterrâneas se intensifica nos grandes centros urbanos, principalmente pelo uso e ocupação do solo, gerando efluentes diversos que retornam para os corpos hídricos, interferindo em sua qualidade, e em menor intensidade pela sazonalidade (AZEVEDO, 2006; CAMPANHA et al., 2010). Por isso, o monitoramento de águas subterrâneas empregando análises químicas é uma medida importante para avaliar a sua qualidade, servindo de indicativos para identificação de possíveis fontes de contaminação, que podem alterar significativamente as propriedades químicas da água, comprometendo o equilíbrio geral do sistema, causando prejuízos econômicos e inviabilizando seu consumo (REBOUÇAS et al., 2006).

Segundo Rebouças et al. (2006), as águas subterrâneas vêm perdendo em qualidade devido à sua contaminação por fossas sépticas, aterros sanitários, contaminação por pesticidas, fertilizantes, intrusão salina, fraturas em oleodutos, poços abandonados ou mal selados, resíduos industriais, depósitos subterrâneos de produtos químicos e vazamento tanques de armazenamento subterrâneo de combustíveis, etc.. A contaminação de aquíferos por diversas fonte de contaminação antropogênicas e/ou naturais vem sendo uma preocupação mundial e tem sido também muito discutida no Brasil (DÓREA et al., 2007; FORTE et al., 2007).

Na região urbana da Cidade de Parauapebas, Estado do Pará, uma grande quantidade de condomínios residências e domicílios particulares utilizam para abastecimento, poços tubulares e escavados do tipo "Amazonas" como fonte de captação de água de mananciais subterrâneos, proveniente de corpo hidrogeológico (CARVALHO et al., 2019). Além disso, estes poços são perfurados, na maioria das vezes, sem levar em consideração os aspectos hidrogeológicos desse manancial e as proximidades de fossas (sépticas ou rudimentares) e esgotos, nem são sempre construídos obedecendo a critérios técnicos recomendados pela Associação Brasileira de Normas Técnicas (ABNT), apresentando grande vulnerabilidade à contaminação (CABRAL, 2007; CARVALHO et al., 2015).

Atualmente no Brasil, existem duas legislações vigentes para avaliação da qualidade da água. A Portaria № 2914/2011 do Ministério da Saúde trata dos procedimentos de controle e de vigilância da qualidade da água subterrânea e superficial para consumo humano, relativas aos padrões de potabilidade (MS, 2011). Por outro lado, a Resolução № 396/2008 do Conselho Nacional do Meio Ambiente estabelece a classificação das águas subterrâneas e as diretrizes ambientais para o seu enquadramento, bem como algumas definições relacionadas à suas características e os padrões estabelecidos conforme o seu uso (CONAMA, 2008).

Considerando a relevância de estudos desta natureza e tendo como base o crescente aumento do 
consumo das águas subterrâneas e com o propósito de se obter uma água de melhor qualidade, o objetivo deste estudo tem como finalidade avaliar a qualidade das águas subterrâneas captadas em poços tubulares potencialmente utilizados para consumo humano pela população de nove bairros localizados na Cidade de Parauapebas (Estado do Pará, Brasil) a partir da caracterização físico-química das mesmas, com o intuito de contribuir para o controle da qualidade de potabilidade das águas subterrâneas de acordo com os limites estabelecidos pelo Ministério da Saúde (Portaria № 2914/2011) e Conselho Nacional de Meio Ambiente (Resolução № 396/2008).

\section{MATERIAIS E MÉTODOS}

\section{Descrição da área de estudo}

O estudo foi conduzido na área urbana da cidade de Parauapebas/PA, localizada ao Norte do Brasil e Sudeste do Estado do Pará, de acordo com as seguintes coordenadas: $6.0675^{\circ} \mathrm{S}$ e $49.9022^{\circ} \mathrm{W}$, como se pode observar na Figura 1, a qual ilustra os limites territoriais do município, onde a área urbana é marcada pelo contraste mais escuro.

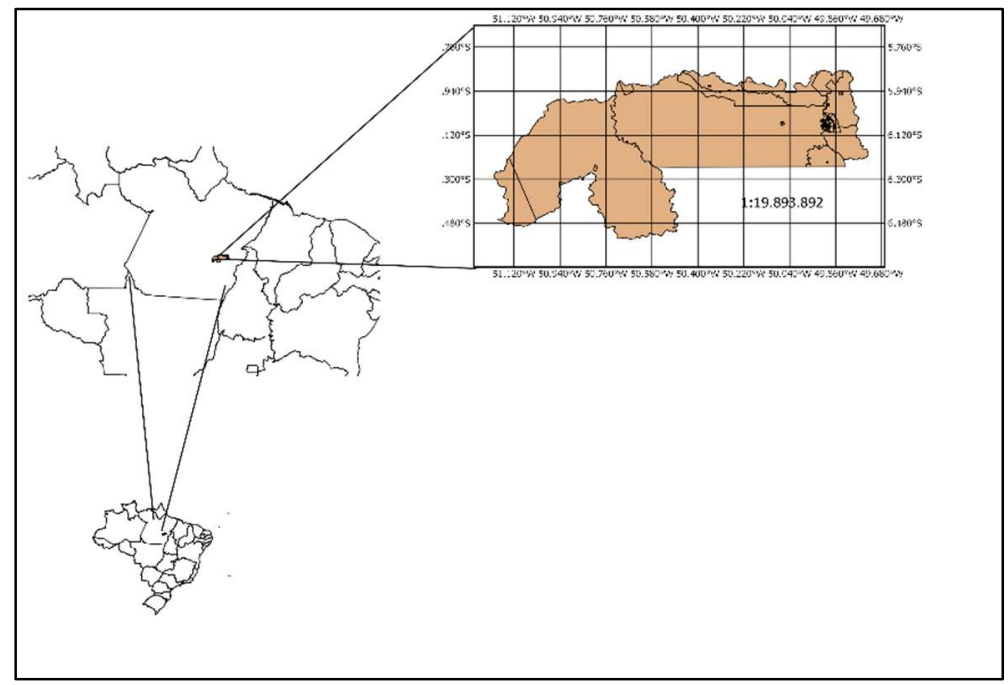

Figura 1: Delimitação territorial do município de Parauapebas.

Inicialmente foi feito um estudo da área, em consonância com a elaboração de um mapa vetorial através do programa Quantum Gis em sua versão 2.18 e com base de dados, referentes as malhas municipais do ano de 2010 (último senso demográfico), fornecidas por meio do site do Instituto Brasileiro de Geografia e Estatística (IBGE). Como os dados obtidos não condiziam com a atualidade, foi elaborado um novo mapa com auxílio do Google Maps e da ferramenta QuickMapServices, presente no software de mapeamento utilizado. Vale ressaltar que os mapas de bairros podem não representar a realidade de forma verossímil, isso, em função da ausência de dados referentes as fronteiras de alguns desses.

Observa-se na Figura 2 a malha urbana do município seguida de um mapa de ampliado para os bairros onde foram realizadas as coletas. A zona ampliada apresenta, quase em sua totalidade, um complexo de bairros centrais, com elevada concentração habitacional e de estabelecimentos 
comerciais, são eles: Da Paz, Rio Verde, Cidade Nova, União, Beira Rio I e II, e Parque dos Carajás I e II; entre as latitudes 603'36"S e 605'24"S, e no intervalo das longitudes 4954'36"W e 4952'12"W.

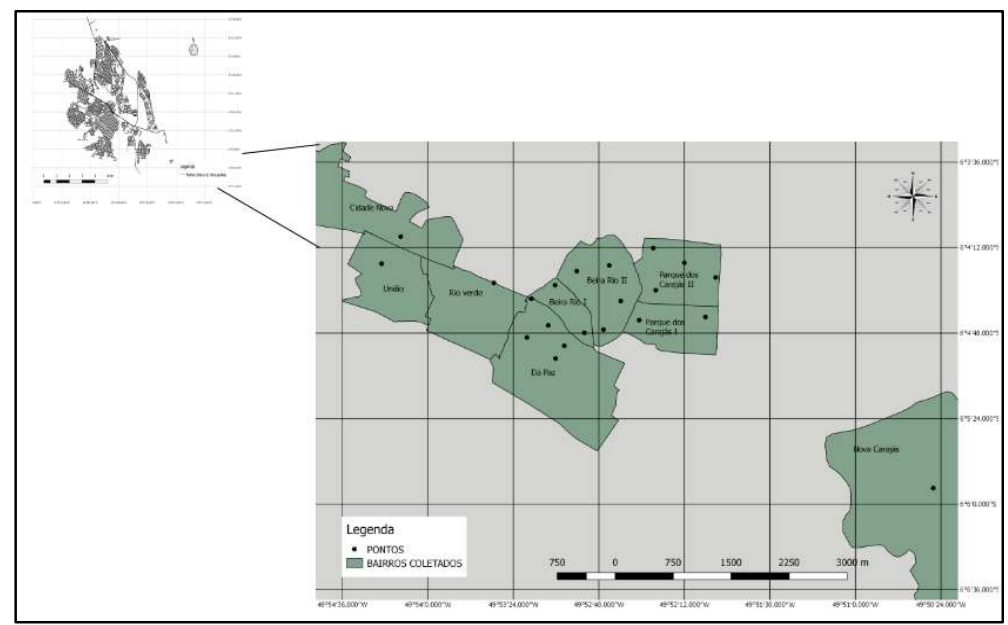

Figura 2: Malha municipal de Parauapebas e ampliação dos respectivos pontos de coleta distribuídos nos nove bairros em estudo.

O maior número de coletas realizadas foi, especialmente nos bairros da Paz, Beira Rio I e II e Parque dos Carajás I e II, devido à predominância de poços tubulares de captação de água subterrânea. A exceção foi o bairro Nova Carajás, que se encontra afastado da região central do município, no qual foi definido apenas um ponto de coleta. Nos bairros União e Rio Verde, por outro lado, as residências consultadas apresentavam, predominantemente, abastecimento da rede pública de água, reduzindo significativamente o número de amostras coletadas nos bairros citados.

Os bairros em estudo estão localizados próximos às microbacias hidrográficas que cortam a cidade, aspecto hidrográfico marcante na cidade de Parauapebas, e por consequência da expansão e desenvolvimento urbano, foram transformados em esgotos a céu aberto, recebendo efluentes domésticos e industriais sem que houvesse algum tratamento prévio destes resíduos (CARVALHO et al., 2015). Todos os pontos de coleta foram georreferenciados utilizando um aparelho de localização (global positioning system, modelo Garmin Map 76) e as localizações obtidas são apresentadas na Tabela 1.

As coletas foram realizadas em residências e condomínios residências horizontais e verticais, que fazem uso de água subterrânea proveniente do lençol freático. Os pontos de coleta, bairros, código de amostras e suas coordenadas geográficas estão descritos de acordo com a tabela 1.

Tabela 1: Coordenadas geográficas dos 20 pontos coletados nos nove bairros da cidade de Parauapebas-PA.

\begin{tabular}{|c|c|c|c|}
\hline Pontos de coleta & Bairro & Código das amostras & Coordenadas geográficas \\
\hline 1 & Rio Verde & RV1 & 604'27,12"S e 49 $53^{\prime} 32,14^{\prime \prime} \mathrm{W}$ \\
\hline 2 & $\mathrm{DaPaz}$ & DP1 & $604^{\prime} 32,97^{\prime \prime}$ S e $49 \circ 53^{\prime} 19,85^{\prime \prime} \mathrm{W}$ \\
\hline 3 & Da Paz & DP2 & 604'49,11"S e 4953'18,39"W W \\
\hline 4 & Da Paz & DP3 & 604'44,62"S e 4953'5,87"W W \\
\hline 5 & $\mathrm{DaPaz}$ & DP4 & 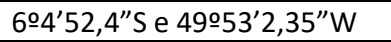 \\
\hline 6 & Da Paz & DP5 & $604^{\prime} 56,2^{\prime \prime}$ S e 4953'6,24"W \\
\hline 7 & União & UN1 & 604'16,64"S e 4954'23,84"W \\
\hline 8 & Cidade Nova & CN1 & 604'4,81"S e 4954'14,04"W W \\
\hline 9 & Nova Carajás & NC1 & $605^{\prime} 52,81^{\prime \prime}$ S e $49 \circ 50^{\prime} 27,41^{\prime \prime} \mathrm{W}$ \\
\hline 10 & Beira Rio I & $\mathrm{BRI}(1)$ & $604^{\prime} 27,12^{\prime \prime}$ S e $49^{\circ} 53^{\prime} 32,14^{\prime \prime} \mathrm{W}$ \\
\hline
\end{tabular}




\begin{tabular}{|c|c|c|c|}
\hline 11 & Beira Rio I & $\mathrm{BRI}(2)$ & 604'32,97"S e 4953'19,85”W \\
\hline 12 & Beira Rio II & BRII(1) & 604'49,11"S e 4953'18,39”W \\
\hline 13 & Beira Rio II & BRII(2) & 604'44,62"S e 4953'5,87"W \\
\hline 14 & Beira Rio II & BRII(3) & $6044^{\prime} 52,4^{\prime \prime}$ S e 4953'2,35”W \\
\hline 15 & Beira Rio II & BRII(4) & 604'56,2"S e 4953'6,24"W \\
\hline 16 & Parque dos Carajás I & $\mathrm{PCl}(1)$ & 604'16,64"S e 4954'23,84"W \\
\hline 17 & Parque dos Carajás I & $\mathrm{PCl}(2)$ & 604'4,81"S e 4954'14,04"W W \\
\hline 18 & Parque dos Carajás II & $\mathrm{PCII}(1)$ & 605'52,81"S e 4950'27,41"W W \\
\hline 19 & Parque dos Carajás II & $\mathrm{PCII}(2)$ & 604'4,81"S e 4954'14,04"W W \\
\hline 20 & Parque dos Carajás II & PCII(3) & $605^{\prime} 52,81^{\prime \prime}$ S e $49 \circ 50$ '27,41"W \\
\hline
\end{tabular}

\section{Amostragem}

Entre os meses de maio e junho de 2019, vinte amostras de água subterrânea foram coletadas nos pontos de amostragem distribuídos em nove bairros da cidade de Parauapebas (Da Paz: 5 amostras; Beira Rio II: 4 amostras; Parque dos Carajás II: 3 amostras; Parque dos Carajás I: 2 amostras; Beira-Rio I: 2 amostras; Cidade Nova: 1 amostra; Nova Carajás: 1 amostra; Rio Verde: 1 amostra e União: 1 amostra).

As amostras foram coletadas em frascos de polietileno $(500 \mathrm{~mL})$ previamente esterilizados para realização das análises físico-químicas. Após a coleta, todas as amostras foram levadas para o Laboratório de Química do Campus de Parauapebas da Universidade Federal Rural da Amazônia devidamente identificadas, mantidas sobre refrigeração à temperatura de $4{ }^{\circ} \mathrm{C}$ e protegidas da luz para realização das análises físicoquímicas. Todos os parâmetros físico-químicos foram realizados em triplicata.

\section{Instrumentos e acessórios}

Um termômetro de mercúrio (marca Incoterm) com escala entre 0 e $50{ }^{\circ} \mathrm{C}$ foi utilizado para medir a temperatura no momento da coleta. As demais análises foram realizadas no laboratório, com phmetro de bancada (Marca Hanna Instruments, Modelo HI9321Q-799D), condutivímetro de bancada (Marca Hanna Instruments, Modelo HMCDB-150), turbidímetro de bancada (Marca Cheeselab, Modelo CL 1000). A cor, o teor de cloreto $\left(\mathrm{Cl}^{-}\right)$e os sólidos totais dissolvidos (STD) foram determinados de acordo com o Standard Methods for the Examination of Water and Wastewater from Americam Public Health Association (APHA, 2005).

Os materiais de plástico de polietileno, vidrarias e frascos volumétricos foram lavados com água corrente, depois com água desionizada e, em seguida foram imersos em banho em solução de $\mathrm{HNO}_{3} 10 \%$ $(\mathrm{v} / \mathrm{v})$ durante $24 \mathrm{~h}$. Posteriormente, esses materiais foram lavados abundantemente com água desionizada e secos a temperatura ambiente.

\section{Determinação dos parâmetros físico-químicos}

Foram analisados os seguintes parâmetros físico-químicos: A temperatura $(T)$, o potencial hidrogeniônico $(\mathrm{pH})$, a condutividade elétrica (CE) e a turbidez (TRB) foram medidas diretamente nas amostras brutas. A cor foi determinada pelo método de comparação visual (Método 2120B). O teor de cloreto ( $\mathrm{Cl}$ ) foi determinado pelo método argentimétrico (Método 4500B). Os sólidos totais dissolvidos (STD) foram determinados pelo método gravimétrico (Método 2540B) (APHA, 2005). Todas as análises foram realizadas em triplicata $(n=3)$, com exceção da temperatura somente uma replicata $(n=1)$. 


\section{Análise estatística dos dados}

Para o tratamento estatístico dos resultados presente neste estudo, foram utilizados recursos básicos de estatística descritiva (média, desvio padrão, valores mínimos e máximos) é o método multivariado de correlação de Pearson, utilizando o software Statistica 8.0 (StatSoft, Inc., Tulsa, OK, USA) para análise dos dados. O nível de significância obtido para a análise estatística dos dados foi de $p<0.05$

Antes da aplicação da análise multivariada, primeiramente foi construído uma matriz de dados contendo 20 linhas (amostras) e 7 colunas (variáveis). 0 tipo de pré-processamento da matriz de dados originais foi o auto-escalonamento (MINGOTI, 2005). Assim, os dados auto-escalados de cada variável terão a média igual a zero e o desvio padrão igual a 1, de forma que todas as variáveis passam a ter a mesma importância, ou seja, o mesmo peso, ocorrendo à normalização dos dados, procedimento que elimina a influência de diferentes unidades de medida (BEEBE et al., 1998). A correlação linear de Pearson foi utilizada para verificar o grau de dependência ou independência de uma variável em relação à outra (MINGOTI, 2005).

\section{RESULTADOS E DISCUSSÃO}

\section{Avaliação da qualidade das águas subterrâneas}

Os valores médios dos resultados obtidos correspondentes aos 7 parâmetros físico-químicos determinados nas amostras de águas subterrâneas estudadas estão apresentados na Tabela 2. Os resultados obtidos foram comparados com os valores padrões indicados pela legislação brasileira de referência, MS 2914/2011 e CONAMA 396/2008, acerca da qualidade de águas potável e subterrânea, respectivamente.

Tabela 2: Valores obtidos para os parâmetros físico-químicos das amostras de águas subterrâneas.

\begin{tabular}{|c|c|c|c|c|c|c|c|c|c|c|c|c|c|c|c|c|c|c|c|c|c|}
\hline $\begin{array}{l}\text { Parâm } \\
\text { etros }\end{array}$ & $\begin{array}{l}\text { Unid } \\
\text { ades }\end{array}$ & $\begin{array}{l}\mathrm{DP} \\
1\end{array}$ & $\begin{array}{l}\mathrm{DP} \\
2\end{array}$ & $\begin{array}{l}\mathrm{DP} \\
3\end{array}$ & $\begin{array}{l}\mathrm{DP} \\
4\end{array}$ & $\begin{array}{l}\mathrm{DP} \\
5\end{array}$ & $\begin{array}{l}\text { BR } \\
\text { II1 }\end{array}$ & $\begin{array}{l}\text { BR } \\
\text { II2 }\end{array}$ & $\begin{array}{l}\text { BR } \\
\| 3\end{array}$ & $\begin{array}{l}\text { BR } \\
\text { II4 }\end{array}$ & $\begin{array}{l}\text { PC } \\
\text { II1 }\end{array}$ & $\begin{array}{l}\mathrm{PC} \\
112\end{array}$ & $\begin{array}{l}\mathrm{PC} \\
\text { II3 }\end{array}$ & $\begin{array}{l}\text { PC } \\
\text { I1 }\end{array}$ & $\begin{array}{l}\mathrm{PC} \\
12\end{array}$ & $\begin{array}{l}\text { BR } \\
\text { I1 }\end{array}$ & $\begin{array}{l}\text { BR } \\
12\end{array}$ & $\begin{array}{l}\mathrm{C} \\
\mathrm{N} \\
1\end{array}$ & $\begin{array}{l}\mathrm{N} \\
\mathrm{C} 2\end{array}$ & $\begin{array}{l}\mathrm{R} \\
\mathrm{V} 1\end{array}$ & $\begin{array}{l}U \\
N \\
1\end{array}$ \\
\hline$T$ & ${ }^{\circ} \mathrm{C}$ & 2 & $\begin{array}{l}26 . \\
5\end{array}$ & $\begin{array}{l}27 . \\
0\end{array}$ & $\begin{array}{l}26 \\
5\end{array}$ & $\begin{array}{l}25, \\
5\end{array}$ & $\begin{array}{l}26 . \\
5\end{array}$ & $\begin{array}{l}26 \\
5\end{array}$ & $\begin{array}{l}27 . \\
0\end{array}$ & $\begin{array}{l}27 . \\
0\end{array}$ & $\begin{array}{l}26 . \\
0\end{array}$ & $\begin{array}{l}26 . \\
5\end{array}$ & $\begin{array}{l}25, \\
0\end{array}$ & $\begin{array}{l}25 \\
, 5\end{array}$ & $\begin{array}{l}26 \\
, 0\end{array}$ & $\begin{array}{l}27 \\
, 0\end{array}$ & $\begin{array}{l}27 \\
.0\end{array}$ & $\begin{array}{l}27 \\
, 0\end{array}$ & $\begin{array}{l}26 \\
.5\end{array}$ & $\begin{array}{l}26 \\
, 0\end{array}$ & $\begin{array}{l}27 \\
, 0\end{array}$ \\
\hline $\mathrm{pH}$ & 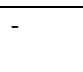 & $\begin{array}{l}5.3 \\
4\end{array}$ & $\begin{array}{l}5,8 \\
5\end{array}$ & $\begin{array}{l}5,2 \\
6\end{array}$ & $\begin{array}{l}5,5 \\
3\end{array}$ & $\begin{array}{l}5,7 \\
2\end{array}$ & $\begin{array}{l}5.6 \\
2\end{array}$ & $\begin{array}{l}6.0 \\
8\end{array}$ & $\begin{array}{l}5,8 \\
2\end{array}$ & $\begin{array}{l}5.9 \\
2\end{array}$ & $\begin{array}{l}5,5 \\
8\end{array}$ & $\begin{array}{l}5.8 \\
3\end{array}$ & $\begin{array}{l}6.1 \\
2\end{array}$ & $\begin{array}{l}6, \\
05\end{array}$ & $\begin{array}{l}5, \\
67\end{array}$ & $\begin{array}{l}5 \\
86\end{array}$ & $\begin{array}{l}5 \\
54\end{array}$ & $\begin{array}{l}5, \\
60\end{array}$ & $\begin{array}{l}5 \\
46\end{array}$ & $\begin{array}{l}5 \\
57\end{array}$ & $\begin{array}{l}5, \\
62\end{array}$ \\
\hline TRB & UNT & 1.5 & 1,5 & $\begin{array}{l}1,2 \\
5\end{array}$ & $\begin{array}{l}1,7 \\
5\end{array}$ & 1,0 & $\begin{array}{l}1.7 \\
5\end{array}$ & 1.5 & $\begin{array}{l}1.2 \\
5\end{array}$ & $\begin{array}{l}1.2 \\
5\end{array}$ & 1.0 & $\begin{array}{l}1.2 \\
5\end{array}$ & 1,5 & $\begin{array}{l}1, \\
5\end{array}$ & $\begin{array}{l}1, \\
5\end{array}$ & $\begin{array}{l}1 . \\
5\end{array}$ & 1. & $\begin{array}{l}1 . \\
25\end{array}$ & $\begin{array}{l}1 . \\
0\end{array}$ & $\begin{array}{l}1, \\
0\end{array}$ & $\begin{array}{l}1, \\
5\end{array}$ \\
\hline $\mathrm{CE}$ & $\begin{array}{l}\mu \mathrm{S} \\
\mathrm{cm}^{-1}\end{array}$ & $\begin{array}{l}16 \\
4,4\end{array}$ & $\begin{array}{l}17 \\
6.3\end{array}$ & $\begin{array}{l}13 \\
6,3\end{array}$ & $\begin{array}{l}15 \\
8.4\end{array}$ & $\begin{array}{l}21 \\
6,7\end{array}$ & $\begin{array}{l}87 . \\
2\end{array}$ & $\begin{array}{l}74 . \\
0\end{array}$ & $\begin{array}{l}72 . \\
2\end{array}$ & $\begin{array}{l}65, \\
5\end{array}$ & $\begin{array}{l}68 . \\
3\end{array}$ & $\begin{array}{l}74 . \\
1\end{array}$ & $\begin{array}{l}60 . \\
2\end{array}$ & $\begin{array}{l}77 \\
.3\end{array}$ & $\begin{array}{l}59 \\
4\end{array}$ & $\begin{array}{l}71 \\
.7\end{array}$ & $\begin{array}{l}55 \\
.0\end{array}$ & $\begin{array}{l}54 \\
.3\end{array}$ & $\begin{array}{l}81 \\
.6\end{array}$ & $\begin{array}{l}51 \\
, 0\end{array}$ & $\begin{array}{l}63 \\
8\end{array}$ \\
\hline Cor & $\mathrm{uH}$ & $\begin{array}{l}10 . \\
0\end{array}$ & 5.0 & 5.0 & $\begin{array}{l}15 . \\
0\end{array}$ & $\begin{array}{l}15 . \\
0\end{array}$ & 5.0 & $\begin{array}{l}10 . \\
0\end{array}$ & 7.5 & $\begin{array}{l}10 . \\
0\end{array}$ & $\begin{array}{l}10 . \\
0\end{array}$ & $\begin{array}{l}10 . \\
0\end{array}$ & 5.0 & $\begin{array}{l}5 . \\
0\end{array}$ & $\begin{array}{l}5, \\
0\end{array}$ & $\begin{array}{l}10 \\
.0\end{array}$ & $\begin{array}{l}10 \\
.0\end{array}$ & $\begin{array}{l}7 . \\
5\end{array}$ & $\begin{array}{l}7 . \\
5\end{array}$ & $\begin{array}{l}10 \\
, 0\end{array}$ & $\begin{array}{l}10 \\
, 0\end{array}$ \\
\hline $\mathrm{Cl}^{-}$ & $\mathrm{mg} \mathrm{L}^{-1}$ & $\begin{array}{l}21 . \\
0\end{array}$ & $\begin{array}{l}17 . \\
4\end{array}$ & $\begin{array}{l}20 . \\
4\end{array}$ & $\begin{array}{l}12 . \\
7\end{array}$ & $\begin{array}{l}26 . \\
1\end{array}$ & $\begin{array}{l}23 . \\
2\end{array}$ & $\begin{array}{l}10, \\
6\end{array}$ & $\begin{array}{l}15 . \\
3\end{array}$ & $\begin{array}{l}11 . \\
8\end{array}$ & $\begin{array}{l}14, \\
1\end{array}$ & $\begin{array}{l}11 . \\
0\end{array}$ & $\begin{array}{l}18 . \\
3\end{array}$ & $\begin{array}{l}15 \\
.5\end{array}$ & $\begin{array}{l}12 \\
, 7\end{array}$ & $\begin{array}{l}12 \\
.2\end{array}$ & $\begin{array}{l}10 \\
.2\end{array}$ & $\begin{array}{l}14 \\
7\end{array}$ & $\begin{array}{l}16 \\
, 2\end{array}$ & $\begin{array}{l}25 \\
, 7\end{array}$ & $\begin{array}{l}16 \\
4\end{array}$ \\
\hline STD & $\mathrm{mg} \mathrm{L}^{-1}$ & $\begin{array}{l}17 \\
7.5\end{array}$ & $\begin{array}{l}20 \\
3.0\end{array}$ & $\begin{array}{l}18 \\
5.5\end{array}$ & $\begin{array}{l}17 \\
5.0\end{array}$ & $\begin{array}{l}27 \\
1,8\end{array}$ & $\begin{array}{l}91 . \\
5\end{array}$ & $\begin{array}{l}83 . \\
0\end{array}$ & $\begin{array}{l}87 . \\
0\end{array}$ & $\begin{array}{l}74 . \\
0\end{array}$ & $\begin{array}{l}76 . \\
0\end{array}$ & $\begin{array}{l}85 . \\
5\end{array}$ & $\begin{array}{l}70 . \\
5\end{array}$ & $\begin{array}{l}88 \\
.5\end{array}$ & $\begin{array}{l}69 \\
, 3\end{array}$ & $\begin{array}{c}77 \\
.5\end{array}$ & $\begin{array}{l}71 \\
.5\end{array}$ & $\begin{array}{l}68 \\
.0\end{array}$ & $\begin{array}{l}79 \\
.0\end{array}$ & $\begin{array}{l}64 \\
, 8\end{array}$ & $\begin{array}{l}56 \\
5\end{array}$ \\
\hline
\end{tabular}

Os valores de estatística descritiva e os valores máximos permitidos (VMP) para os parâmetros físicoquímicos determinados nas amostras de águas subterrâneas estão apresentados na Tabela 3. Os valores obtidos de temperatura em todas as amostras variaram de 25 a $27^{\circ} \mathrm{C}$, apresentando uma amplitude térmica pequena, isto é, as temperaturas nas águas não sofreram grandes mudanças (DIAS et al., 2004).

Tabela 3: Valores de estatística descritiva e VMP para todos os parâmetros físico-químicos determinados nas amostras de água subterrânea $(N=21)$.

\begin{tabular}{|l|l|l|l|l|l|}
\hline Parâmetros & Unidades & Média \pm SD & Mínimo - Máximo & MS 2914/2011 a & CONAMA \\
\hline
\end{tabular}




\begin{tabular}{|l|l|l|l|l|l|}
\hline & & & & & $396 / 2008^{\mathrm{a}}$ \\
\hline \multirow{2}{*}{$\mathrm{T}$} & $\mathrm{pH}$ & $26,45 \pm 0,46$ & $25,0-27,0$ & - & - \\
\hline & - & $5,70 \pm 0,82$ & $5,26-6,12$ & 6,0 a $9,5^{1-}$ & - \\
\hline $\mathrm{TRB}$ & $\mathrm{UNT}$ & $1,33 \pm 0,36$ & $1,0-1,75$ & 5 & - \\
\hline $\mathrm{CE}$ & $\mathrm{\mu S} \mathrm{cm}-1$ & $93,38 \pm 45,2$ & $51,0-216,7$ & - & - \\
\hline Cor & $\mathrm{uH}$ & $8,62 \pm 2,76$ & $5,0-15,0$ & 15 & - \\
\hline $\mathrm{Cl}$ & $\mathrm{mg} \mathrm{L}-1$ & $16,27 \pm 6,35$ & $10,2-26,1$ & 250 & 250 \\
\hline STD & $\mathrm{mg} \mathrm{L}^{-1}$ & $107,80 \pm 64,6$ & $56,5-271,8$ & 1000 & 1000 \\
\hline
\end{tabular}

$\mathrm{SD}=$ desvio-padrão; ${ }^{\mathrm{a}}$ valores máximos permitidos (VMP) e ${ }^{1}$ faixa recomendada pela Portaria № 2914/2011 do MS.

O valor de pH nas amostras variou de 5,26 a 6,12, observando-se que na área de estudo temos águas com características predominantemente ácidas. Somente as amostras BRII2, PCl1 e PCII3 obtiveram pH de acordo com o valor recomendado pelo MS 2914/2011 (6,0 a 9,5), enquanto as demais amostras o pH variou de 5,26 a 5,92, sendo consideradas águas levemente ácidas, refletindo a acidez característica das águas subterrâneas da região (AZEVEDO, 2006; CABRAL et al., 2006). De acordo com Carvalho et al. (2015), águas subterrâneas que apresentam pH ácido pode ser indício de contaminação dos poços tubulares por fossas e esgotos domésticos. Vale ressaltar que o pH das águas naturais influencia na solubilidade de compostos contendo metais traços, com a ordem decrescente de solubilidade, $\mathrm{Cd}>\mathrm{Cu}>\mathrm{Pb}$, sendo esta fração solúvel e biodisponível para interagir com organismos vivos (FONTENELLE et al., 2009).

Os valores de condutividade elétrica variaram de 51,0 a $216,7 \mu \mathrm{S} \mathrm{cm}^{-1}$ e encontram-se abaixo dos teores obtidos em estudos de outros autores (AZEVEDO, 2006; CABRAL et al., 2006; CARVALHO et al., 2015). Todas as amostras de água subterrâneas coletadas no bairro Da Paz apresentaram CE $>100 \mu \mathrm{S} \mathrm{cm}^{-1}$, indicando altos teores de sais dissolvidos ionizados, podendo as mesmas estarem contaminadas decorrentes do despejo de efluentes domésticos, com isso aumentando o grau de salinidade destas águas (SANTOS et al., 2011).

Os valores obtidos de teor de cloreto $\left(10,2\right.$ a $\left.25,7 \mathrm{mg} \mathrm{L}^{-1}\right)$, sólidos totais dissolvidos $(56,5$ a $271,8 \mathrm{mg}$ $\mathrm{L}^{-1}$ ) e turbidez (1,0 a 1,75 NTU), encontram-se abaixo dos valores máximos permitidos pela MS 2914/2011 e CONAMA 396/2008. Porém, as amostras com teores mais elevados de sólidos totais dissolvidos e condutividade elétrica estão fortemente associadas à contaminação pela descarga de efluentes domésticos (SANTOS et al., 2011). A cor variou de 5,0 a 15,0 $\mu \mathrm{H}$, mas somente as amostras DP4 e DP5 coletadas no bairro Da Paz apresentaram valores iguais ao limite máximo permitido $(15,0 \mu \mathrm{H})$ pela MS $2914 / 2011$. Isto ocorre porque estas amostras sofrem influência da maior carga de material sólido em suspensão (BAIG et al., 2009).

\section{Matriz de correlação de Pearson}

Avaliando os dados obtidos através das correlações de Pearson, podem ser observadas correlações positivas fortes entre as variáveis físico-químicas STD $\times$ CE $(r=0,96 ; p<0,01)$, STD $\times$ TRB $(r=0,91 ; p<0,01)$, STD $\times \mathrm{Cl}^{-}(r=0,82 ; p<0,01)$ e consequentemente entre CE X TRB $(r=0,91 ; p<0,01), \mathrm{CE} \times \mathrm{Cl}^{-}(r=0,76 ; p<$ $0,01)$, TRB $\times \mathrm{Cl}^{-}(r=0,68 ; p<0,01)$. De acordo com os estudos de Conceição et al. (2010) e Carvalho et al. (2015), correlações significativas encontradas entre estas variáveis estão diretamente associadas com a contribuição da descarga de efluentes domésticos e características minerais das aguas subterrâneas da 
região em estudo. Uma correlação negativa forte foi observada entre $\mathrm{Cor} \times \mathrm{Cl}^{-}(r=0,80 ; p<0,01)$

\section{CONCLUSÕES}

Os resultados obtidos para todos os parâmetros físico-químicos estudados mostraram que todas as amostras de águas subterrâneas atenderam os limites estabelecidos pela Portaria MS 2914/2011 e Resolução CONAMA 396/2008, não caracterizando um potencial risco para a saúde da população dos bairros da Cidade de Parauapebas onde foram realizadas coletas de águas subterrâneas que fazem uso de poços tubulares para captação de águas subterrâneas para consumo humano. Porém, após a avaliação dos resultados mais elevados de condutividade elétrica, sólidos totais dissolvidos e cor, para as amostras de águas subterrâneas coletadas no bairro Da Paz, servindo de indicativos para identificação de possíveis atividades antropogênicas de contaminação, principalmente em relação à descarga de efluentes domésticos.

AGRADECIMENTOS: ao Conselho Nacional de Desenvolvimento Científico e Tecnológico (CNPq, Brasília, Brasil) pela bolsa de iniciação científica concedida ao discente Henrique Souza Chaves (Edital PROPED no 04/2018).

\section{REFERÊNCIAS}

APHA. American Public Health Association. Standard Methods for the Examination of Water and Wastewater. American Water Works Association (AWWA) and Water Environment Federation (WEF). 21 ed. Washington: APHA, 2005.

AZEVEDO, R. P.. Uso de água subterrânea em sistema de abastecimento público de comunidades na várzea da Amazônia central. Acta Amazonica, v.36, n.3, p.312-320, 2006. DOI: http://doi.org/10.1590/S0044$\underline{59672006000300004}$

BAIG, J. A.; KAZI, T. G.; ARAIN, M. B.; AFRIDI, H. I.; KANDHRO, G. A.; SARFRAZ, R. A.; JAMAL, M. K.; SHAH, A. Q.. Evaluation of arsenic and other physicochemical parameters of surface and groundwater of Jamshoro, Pakistan. Journal of Hazardous Materials, v.166, p.662-669, 2009. DOI: http://doi.org/10.1016/j.jhazmat.2008.11.069

BEEBE, K. R.; PELL, R. J.; SEASHOLTZ, M. B.. Chemometrics: A Pratical Guide. New York: John Wiley \& Sons, 1998.

CABRAL, N. M. T.. Teores de (NO3-) e amônio $\left(\mathrm{NH}^{+}\right)$nas águas do aquífero Barreiras nos bairros do Reduto, Nazaré e Umarizal - Belém/PA. Química Nova, v.30, n.8, p.1804-1808, 2007. DOI: http://doi.org/10.1590/S010040422007000800003

CABRAL, N. M. T.; LIMA, L. M.. Comportamento hidrogeoqímico das águas do aquífero Barreiras nos bairros centrais de Belém, Pará. Boletim do Museu Paraense Emílio Goeldi. Ciências Naturais, v.1, n.1, p.149-166, 2006.

CAMPANHA, M. B.; MELO, C. A.; MOREIRA, A. B.; FERRARESE, R. F. M. S.; TADINI, A. M.; GARBIN, E. V.;
BISINOTI, M. C.. Variabilidade espacial e temporal de parâmetros físico-químicos nos rios turvo, preto e grande no Estado de São Paulo, Brasil. Química Nova, v.33, n.9, p.18311836, 2010. DOI: http://doi.org/10.1590/S0100$\underline{40422010000900002}$

CARVALHO, F. I. M.; DANTAS FILHO, H. A.; DANTAS, K. G. F.. Simultaneous determination of 16 polycyclic aromatic hydrocarbons in groundwater by GC-FID after solid-phase extraction. SN Applied Sciences, v.1, p.804, 2019. DOI: http://doi.org/10.1007/s42452-019-0839-z

CARVALHO, F. I. M.; LEMOS, V. P.; DANTAS FILHO, H. A.; DANTAS, K. G. F.. Assessment of Groundwater Quality from the Belém Based on Physicochemical Parameters and Levels of Trace Elements Using Multivariate Analysis. Revista Virtual de Química, v.7, n.6, p.2221-2241, 2015. DOI: http://doi.org/10.5935/1984-6835.20150132

CONAMA. Conselho Nacional de Meio Ambiente. Resolução n. 396 de 3 de abril de 2008. Brasília: DOU, 2008.

CONCEIÇÃO, F. T.; SARDINHA, D. S.; SOUZA, A. D. G.; NAVARRO, G. R. B.. Anthropogenic influences on annual flux of cations and anions at meio stream basin, São Paulo State, Brazil. Water, Air, and Soil Pollution, n.205, p.79-91, 2010. DOI: http://doi.org/10.1007/s11270-009-0057-1

DIAS, J. C.; LIMA, W. N.. Comparação de métodos para a determinação de matéria orgânica em amostras ambientais. Revista Científica da UFPA, v.4, p.1-16, 2004.

DÓREA, H. S.; BISPO, J. R. L.; ARAGÃO, K. A. S.; CUNHA, B. B.; NAVICKIENE, S.; ALVES, J. P. H.; ROMÃO, L. P. C.; GARCIA, C. A. B.. Analysis of BTEX, PAHs and metals in the oilfield 
produced water in the State of Sergipe, Brazil.

Microchemical Journal, v.85, p.234-238, 2007. DOI:

http://doi.org/10.1016/j.microc.2006.06.002

FONTENELLE, A. P. G.; PEDROTTI, J. J.; FORNARO, A.. Avaliação de metais traços e íons majoritários em águas subterrâneas na cidade de São Paulo. Química Nova, v.32, n.4, p.839-844, 2009. DOI: http://doi.org/10.1590/S0100$\underline{40422009000400003}$

FORTE, E. J.; AZEVEDO, M. S.; OLIVEIRA, R. C.; ALMEIDA, R.. Contaminação de aquífero por hidrocarbonetos: Estudo de caso na Vila Tupi, Porto Velho - Rondônia. Química Nova, v.30, n.7, p.1539-1544, 2007. DOI:

http://doi.org/10.1590/S0100-40422007000700008

MINGOTI, S. A.. Análise de Dados através de Métodos de Estatística Multivariada. Belo Horizonte: UFMG, 2005.
MS. Ministério da Saúde. Portaria n. 2914 de 12 de dezembro de 2011. Brasília: DOU, 2011.

PANERO, F. S.; SILVA, H. E. B.. Application of exploratory data analysis for the characterization of tubular wells of the North of Brazil. Microchemical Journal, v.88, p.194-200, 2008. DOI: http://doi.org/10.1016/j.microc.2007.11.020

REBOUÇAS, A. C.; BRAGA, B.; TUNDISI, J. G.. Águas doces no Brasil: capital ecológico, uso e conservação. 3 ed. São Paulo: Escrituras, 2006.

SANTOS, J. S.; SANTOS, M. L. P.; ALEXANDRINO, D. M.. Comparative study of the salinalization process in surface water reservoirs located in two distinct regions in southwestern Bahia, Brazil. Journal of the Brazilian Chemical Society, v.22, n.8, p.1418-1425, 2011. DOI: http://doi.org/10.1590/S0103-50532011000800004

A CBPC - Companhia Brasileira de Produção Científica (CNPJ: 11.221.422/0001-03) detém os direitos materiais desta publicação. Os direitos referem-se à publicação do trabalho em qualquer parte do mundo, incluindo os direitos às renovações, expansões e disseminações da contribuição, bem como outros direitos subsidiários. Todos os trabalhos publicados eletronicamente poderão posteriormente ser publicados em coletâneas impressas sob coordenação da Sustenere Publishing, da Companhia Brasileira de Produção Científica e seus parceiros autorizados. Os (as) autores (as) preservam os direitos autorais, mas não têm permissão para a publicação da contribuição em outro meio, impresso ou digital, em português ou em tradução. 